\title{
M-mode echovenography: a new technique for the evaluation of venous wall and valve motion
}

\author{
Jager, K ; Seifert, H ; Bollinger, A
}

\begin{abstract}
The M-mode technique which is widely used in echocardiography allows continuous recording of spontaneous venous wall movements. Compliance of the vein can be quantified. The diameter of the normal vein changes with respiration, while only minor changes are induced by cardiac function. Distensibility and compressibility of the common femoral vein (CFV) were documented. During Valsalva manoeuvre the mean diameter of the CFV increased from 1.05(SD 0.18) $\mathrm{cm}$ to $1.52(0.25) \mathrm{cm}(\mathrm{p}<0.01)$ in the recumbent position and from $1.50(0.20) \mathrm{cm}$ to $1.63(0.17) \mathrm{cm}(\mathrm{NS})$ in the upright position. A patent vein can be completely compressed by the scan head, a thrombosed vein is incompressible. The valve of the normal subclavian vein describes a characteristic M-shaped tracing. The waveform is modulated by cardiac and respiratory function. The study of venous wall movement and venous valve motion provides new insights into venous physiology
\end{abstract}

DOI: https://doi.org/10.1093/cvr/23.1.25

Posted at the Zurich Open Repository and Archive, University of Zurich ZORA URL: https://doi.org/10.5167/uzh-155205

Journal Article

Published Version

Originally published at:

Jager, K; Seifert, H; Bollinger, A (1989). M-mode echovenography: a new technique for the evaluation of venous wall and valve motion. Cardiovascular Research, 23(1):25-30.

DOI: https://doi.org/10.1093/cvr/23.1.25 


\title{
M-mode echovenography: a new technique for the evaluation of venous wall and valve motion
}

\author{
KURT JÄGER,* HILDEGARD SEIFERT, ALFRED BOLLINGER \\ From the Department of Internal Medicine, Angiology Divisions, Kantonsspital, Basel* and the University \\ Hospital, Zürich
}

ABSTRACT The M-mode technique which is widely used in echocardiography allows continuous recording of spontaneous venous wall movements. Compliance of the vein can be quantified. The diameter of the normal vein changes with respiration, while only minor changes are induced by cardiac function.

Distensibility and compressibility of the common femoral vein (CFV) were documented. During Valsalva manoeuvre the mean diameter of the CFV increased from 1.05(SD 0.18) $\mathrm{cm}$ to $1.52(0.25)$ $\mathrm{cm}(\mathrm{p}<0.01)$ in the recumbent position and from $1.50(0.20) \mathrm{cm}$ to $1.63(0.17) \mathrm{cm}(\mathrm{NS})$ in the upright position. A patent vein can be completely compressed by the scan head, a thrombosed vein is incompressible. The valve of the normal subclavian vein describes a characteristic $\mathbf{M}$-shaped tracing. The waveform is modulated by cardiac and respiratory function.

The study of venous wall movement and venous valve motion provides new insights into venous physiology.

Ultrasound has been used in vascular diagnosis for more than 20 years. ${ }^{1-3}$ The transmitted and the reflected sound waves are characterised by their frequency and their amplitude. Instruments detecting frequency of the ultrasonic waves are based on the Doppler principle, while instruments analysing amplitude provide an $\mathrm{A}$-mode $(\mathrm{A}=$ amplitude) or a B-mode image ( $\mathrm{B}=$ brightness), or an $\mathrm{M}$-mode tracing $\left(\mathrm{M}=\right.$ motion) ${ }^{4}{ }^{5} \mathrm{~B}$-mode imaging combined with pulsed Doppler devices corresponds to the duplex technique, ${ }^{6}$ providing anatomical information about the vascular structure and physiological information on blood flow. The $\mathrm{M}$-mode technique presents moving tissue interfaces as a function of time in a one dimensional manner. In echocardiography this technique is routinely used to assess the motion of the myocardium and of the cardiac valves. ${ }^{7}$

The purpose of this paper is to document the value of the M-mode technique in phlebology. We use the

Address for correspondence and reprints: $\mathrm{Dr} K$ Jäger, Department of Internal Medicine, Angiology Division, Kantonsspital, CH-4031 Basel, Switzerland.

Key words: venous diameter changes; venous physiology; distensibility/compressibility of veins; duplex-scan.

Submitted 16 May 1988

Accepted 18 August 1988
M-mode in combination with an imaging unit for registration and quantitative analysis of venous wall motion and venous valve movement.

\section{Methods}

Twenty healthy subjects without venous disease were examined. The mean age was 40.4 years with a range from 21 to 74 years. Six were men and 14 women. A duplex system (Ultra Imager, Biosound, Indianapolis) was used to investigate the subclavian and femoral vein. The $5 \mathrm{MHz}$ transducer was used to generate a two dimensional sector image of the vessel. The B-mode image is used to identify the vessel, to delineate the vessel wall and to depict the venous valves. When the B-Mode image is frozen, the crystal is used either in the pulsed Doppler mode or in the M-mode. The pulsed Doppler device allows accurate analysis of flow within defined regions of the vein. The $\mathrm{M}$-mode tracing provides continuous recordings of moving tissue interfaces as a function of time. The position of the incident ultrasound beam used for both the Doppler and the M-mode analysis is adjustable and displayed on the B-mode image as a white line. In time-motion studies the axis of insonation was perpendicular to the vessel axis. Stationary structures are visualised as straight lines, while moving 
structures such as the venous wall or the venous valves inscribe a wavy line on the echovenogram. Waveforms are displayed graphically with time on the horizontal axis and distance on the vertical axis. The internal dimensions of the vein and the excursions of the valves were measured on the videoscreen with the implemented software.

To form an image of the subclavian vein and its proximal valve, the ultrasonic transducer was placed in the supraclavicular area. In the groin the transducer was positioned in a sagittal plane above the bifurcation of the palpable femoral artery. Then the scan head was gradually moved to the common femoral vein located more medially. A slight movement in the distal direction allows one to visualise the junction of the deep and the superficial femoral veins. In healthy subjects the valve in the proximal part of the superficial femoral vein can be depicted.

With the subject at rest $(>15 \mathrm{~min})$ in a recumbent position the luminal diameters of the subclavian and the common femoral vein were recorded and the spontaneous changes documented on videotape and on hard copy outputs. Changes induced by Valsalva manoeuvre or external compression were evaluated at the level of the common femoral vein. In addition the motion of the proximal valve of the subclavian vein and of the superficial femoral vein were assessed. ECG and respiration were recorded simultaneously.

Results are given as means (SD).

\section{Results}

SPONTANEOUS VENOUS WALL MOTION

Blood vessel walls are readily imaged and well delineated by the $\mathrm{M}$-mode technique. This allows accurate measurements of venous diameter. The diameter of the subclavian vein fluctuates (fig $1 \mathrm{a}$ and b). Phasic changes of internal dimensions occur in
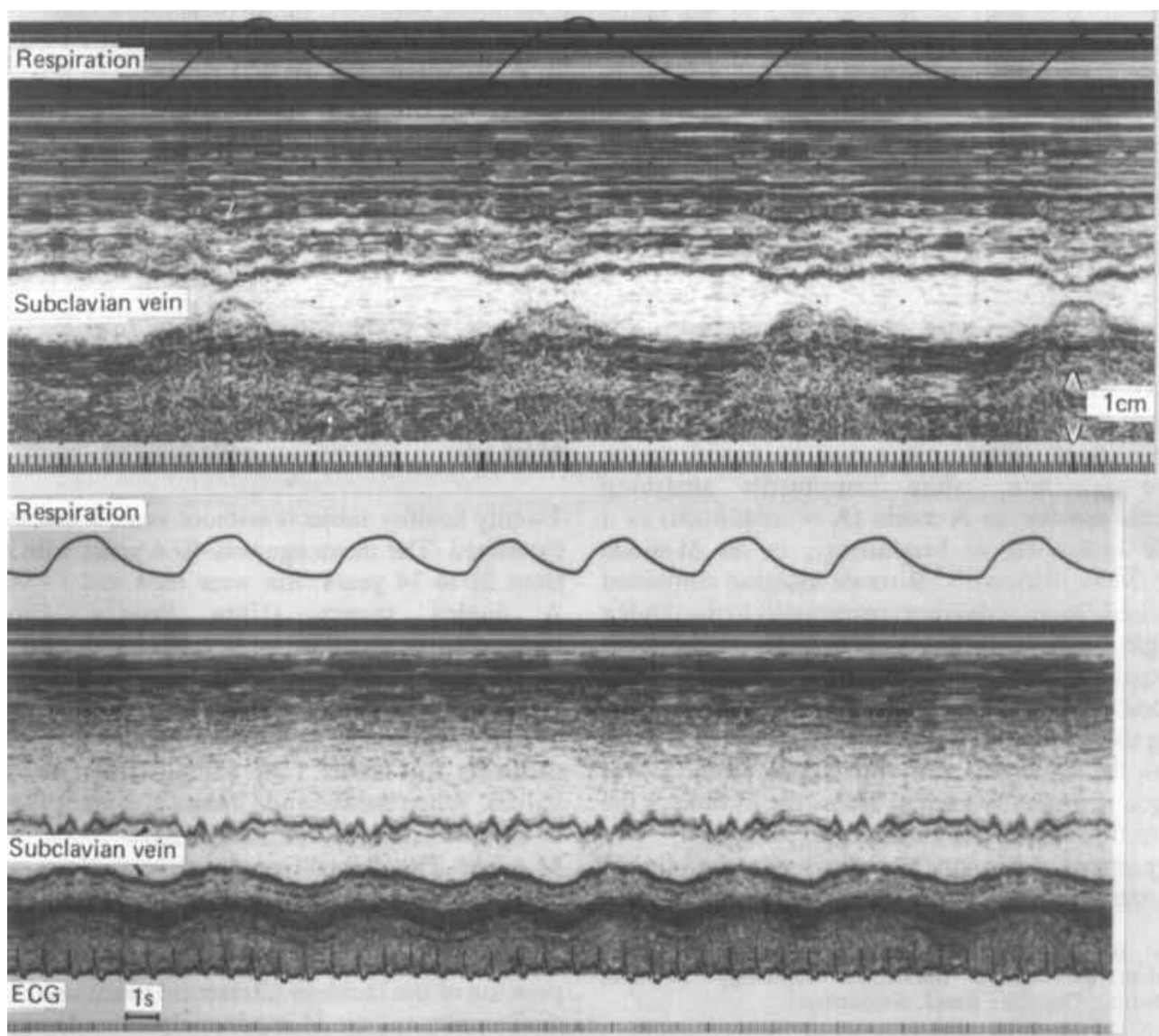

Ho 1 M-mode echovenography of the subclavian vein. Spontaneouschanges in diameter. Upper trace (a): At the end of inspiration the diameter is reduced and the vein tends to collapse. Small cardiac pulsations are superimposed on respiratory modulation of diameter. Lower trace (b): Venous wall pulsations in response to cardiac function are most pronounced during inspiration. 
response to respiration. During expiration the subclavian vein reaches its maximal diameter, averaging $0.8(0.3) \mathrm{cm}$. During inspiration the diameter tends to decrease and reaches a minimal cross section at the end of inspiration (fig 1a). Occasionally the vein collapses completely at the end of inspiration.
Superimposed on respiratory movements of the venous wall, venous pulsations can be documented. They are less pronounced and their higher frequency coincides with heart rate (fig la and b). During inspiration these wall movemens exhibit increased amplitude (fig 1b.).

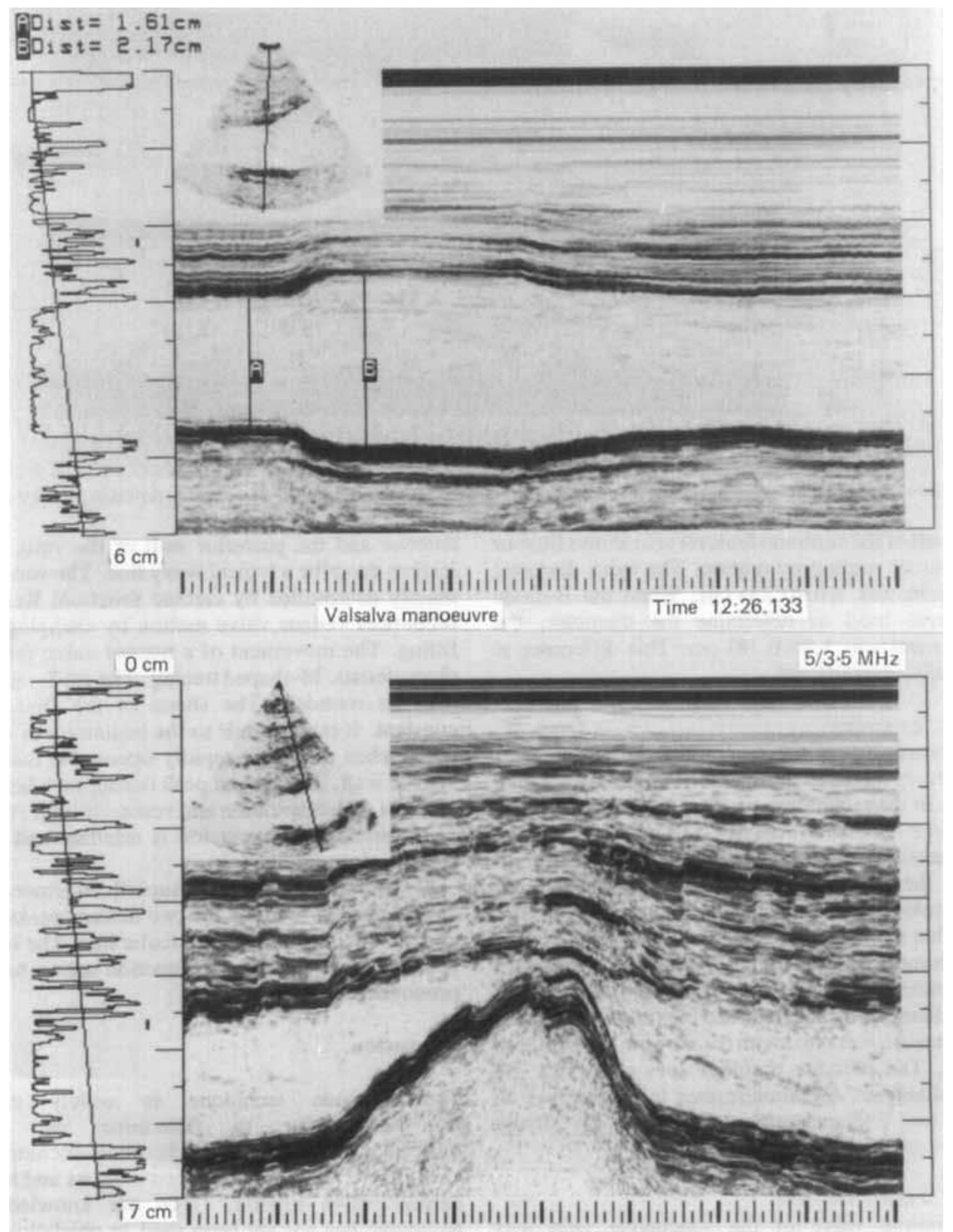

FIG 2 Induced changes in venous diameter. Upper trace (a): Distensibility of the femoral vein during Valsalva manoeuvre $A$ : Diameter at rest. B. Diameter during Valsalva manoeuvre. Lower trace $(b)$ : The normal vein can be compressed completely by a slight external pressure. 


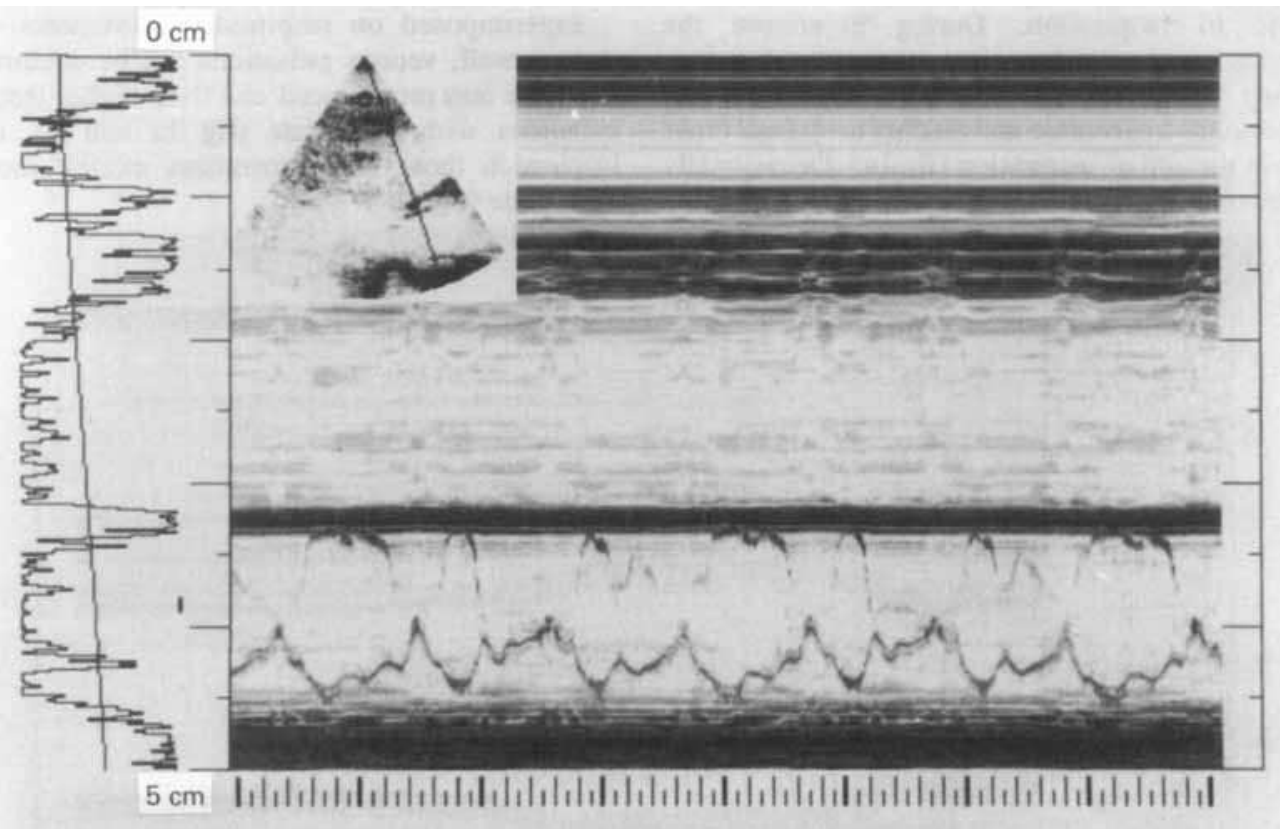

Thi 3 Venous valve motion. Spontaneous movement of subclavian valves is modulated by cardiac function and respiration.

The wall of the common femoral vein shows little or no cardiac or respiratory motion. The mean diameter of the vein was $1.03(0.23) \mathrm{cm}$. When the $\mathrm{B}$-mode image was used to determine the diameter, the measurement was $1.05(0.18) \mathrm{cm}$. This difference is statistically insignificant.

\section{INDUCED CHANGES}

The distensibility of the common femoral vein was continuously recorded during the Valsalva manoeuvre (fig 2a). In the recumbent position the mean diameter increased to $1.52(0.25) \mathrm{cm}(\mathrm{p}<0.01)$. On the average the diameter increased by $44.5(15.0) \%$. In the upright position the mean diameter of the common femoral vein increased from $1.50(0.20) \mathrm{cm}$ to $1.63(0.17) \mathrm{cm}$ (NS). This increase corresponds to $8.6(10) \%$.

The compressibility of the common femoral vein was documented. In the $\mathbf{M}$-mode the reduction in venous diameter can be recorded continuously while a slight pressure is exerted with the ultrasonic transducer (fig 2b). The pressure required does not affect the arterial diameter. An uninterrupted tracing proves an artefact free documentation. A normal vein can be easily compressed by the scan head.

VENOUS VALVE MOTION

The proximal valve of the subclavian vein was visualised on the B-mode image. Usually two long leaflets were seen. The movement of the valves was analysed by $\mathrm{M}$-mode recordings (fig 3 ). Between the anterior and the posterior wall of the vein, the two leaflets describe a typical wavy line. The waveform is mainly determined by cardiac function. Respiration influences venous valve motion by changing venous filling. The movement of a normal valve produces a characteristic M-shaped tracing. The crests taper off or may be rounded. The shape of the first peak is constant. It corresponds to the beginning of the heart cycle when the valve rapidly opens and touches the venous wall. The second peak during each heart cycle changes with respiration and venous filling. At the end of inspiration the excursion is minimal and the two leaflets close completely.

The proximal valve of the superficial femoral vein is also in motion. Usually the two distinct peaks are lost and the tracing shows one circular line. The influence of respiration and cardiac function seems to be less pronounced.

\section{Discussion}

The M-mode technique is widely used in echocardiography to determine the internal dimensions of the cardiac chambers including their changes during myocardial contractions and to assess cardiac valve motion. ${ }^{4}$ To our knowledge this technique has not yet been used in phlebology. This study demonstrates that $\mathrm{M}$-mode recordings in combination with a B-mode imaging unit are suitable for the analysis of venous wall and valve motion. The 
B-mode image provides an excellent two dimensional resolution of tissue interfaces. ${ }^{6-12}$ Relative movements between structures can be visualised and recorded on videotape. A frozen image, however, is necessary for the measurement of dimensions. This measurement represents the immediate situation at the time when the video image was stopped or the photograph was taken from the screen respectively. Movements and relative changes between different tissue structures are not reflected by these measurements. Uninterrupted documentation is only possible by using the M-mode technique. Diameter changes of veins are recorded on hard copy outputs or on videotape. The comparison of the tracings with respiration curves and ECG allows differentiation between the changes induced by respiration or cardiac action.

Spontaneous movements of the venous wall were marked in the subclavian vein and minor in the common femoral vein. Since these movements reflect the influences of cardiac and respiratory cycles the vicinity of the subclavian vein to the heart explains the differences between the two sites of observation. Large changes of venous calibre have been described by invasive catheter techniques. ${ }^{13}$ The authors found a collapse of the vena cava near the diaphragm. As the results of this study demonstrate, venous diameters more distant from the thorax and below the diaphragm fluctuate only to a minor extent. Parallel to these small wall movements, changes of pressure related to respiration or cardiac cycle are dampened in the femoral vein when compared to the subclavian vein or the right atrium. ${ }^{13}$

M-mode echovenography allows assessment of the compliance of the venous wall. The elasticity of the normal vein was evaluated under different conditions such as external compresion and Valsalva manoeuvre. A change in body position from quiet lying to unsupported standing induced a significant increase in common femoral vein diameter of $43 \%$. Almost the same increase in diameter (44\%) was found during Valsalva manoeuvre in the recumbent position. During the Valsalva manoeuvre femoral vein pressure reaches values of $40-90 \mathrm{~mm} \mathrm{Hg} .{ }^{13}$ These pressures cause maximal physiological distension of the veins proximal to the first normal valve. In the upright position the veins are submaximally filled and Valsalva manoeuvre induced an insignificant enlargement of $8 \%$. Distal to a normal valve only minimal changes were detected. First experience in patients suggests that the same holds true for the venous segment proximal to incompetent valves. Additional differentiation of valvular competence or incompetence is possible using the pulsed Doppler unit of the duplex system.

Recently, ultrasound imaging has been used to visualise venous thrombi ${ }^{14}$ supplementing conventional Doppler techniques for early diagnosis.${ }^{15}$ One of the criteria used is the elasticity of the vein. ${ }^{14}$ The normal vein can easily be compressed by a slight external pressure while the thrombosed venous segment remains incompressible. It is obvious that the B-mode image is best suited for visualisation of the induced changes in dimensions of the vein. Continuous recordings of the true changes, proving at the same time that the documentation is free of artefacts, are only possible using the M-mode technique. Therefore, this procedure adds useful potential information for detection of thrombi.

The motion of the proximal subclavian valve may be visualised by duplex scanning and recorded by the M-mode technique (fig 3). The waveform is M-shaped. The two peaks correspond to the opening of the valve when the venous pressure distal to the two leaflets exceeds the proximal pressure. It is known that pressure curves in the superior vena cava or in the deep jugular vein show two peaks. During the $A$ wave of atrial contraction the valves are closed; during the $\mathrm{V}$ wave of passive atrial filling the leaflets move towards centre stream, inducing the incision of the M-shaped tracing. The valves open in response to the $X$ and $Y$ descent of the pressure curve. Flow curves recorded by the Doppler technique are modulated by this movement of the valve. ${ }^{16}$ In healthy subjects the valve closes in midstream position at the end of each heart beat, especially at the end of inspiration. The amplitude of the leaflet movement is determined by respiration and probably by flow rate. The similarity of this tracing to that of a moving mitral valve ${ }^{7}$ is striking.

At the femoral level the movements of the valves are attenuated in agreement with less pronounced wall movements. Both typical peaks of the M-mode tracings are flattened. The small pressure fluctuations in the femoral veins ${ }^{13}$ are probably responsible for this observation.

Since valvular competence is an essential function of the venous system, the new technique yields potential information regarding haemodynamics of leaking valves. $\mathrm{M}$-mode echovenography provides a new way to study physiology and pathophysiology of venous wall and valve motion. It is of potential value for analysing changes induced by valvular insufficiency and venous thrombosis.

\section{References}

1 Satumora $S$. Study of flow patterns in peripheral arteries by ultrasonics. J Acoust Soc Jpn 1959;15:151-8.

2 Rushmer RF, Baker DW, Stegall HF. Transcutaneous Doppler flow detection as a nondestructive technique. $J A p p l$ Physiol 1966;21:554-66.

3 Strandness DE Jr, McCutcheon EP, Rushmer RF. Application of a transcutaneous Doppler flowmeter in the 
evaluation of occlusive arterial disease. Surg Gynecol Obster 1966;122: $1039-45$.

4 Reneman RS. Cardiovascular applications of ultrasound. Amsterdam: North-Holland Publishing, 1974.

5 Milnor WR. Hemodynamics. Baltimore, London: Williams and Wilkins, 1982

6 Phillips DJ, Strandness DE Jr. Duplex scanning. Practica aspects of instrument performance. In: Bernstein EF, ed. Noninvasive diagnostic techniques in vascular disease. St Louis, Toronto, Princeton: CV Mosby, 1985:397-408

7 Feigenbaum .. H. Echocardiography (4th Edition) Philadelphia: Lea and Febiger, 1986.

8 Comerota AJ, Cranley JJ, Hyden WG. Ultrasonic imaging for carotid occlusive disease, In: Bernstein EF, ed. Noninvasive diagnostic techniques in vascular disease. S Louis, Toronto, Princeton: CV Mosby, 1985:384-96.

9 Roederer GO; Langlois YE, Jäger KA, et al. The natural history of carotid arterial disease in asymptomatic patients with cervical bruits. Stroke 1984;15:605-13.

10 Jäger KA, Ricketts HJ, Strandness DE Jr. Duplex scanning for the evaluation of lower limb arterial disease. In: Bernstein EF. ed. Noninvasive diagnostic techniques in vascular disease. St Louis, Toronto, Princeton: CV Mosby, 1985:619-31.

11 Jäger K, Bollinger A, Valli C, Ammann R. Measurement of mesenteric blood flow by duplex scanning. $J$ Vasc Surg 1986;3:462-9.

12 Kohler TR, Zierler RE, Martin RL, et al. Noninvasive diagnosis of renal artery stenosis by ultrasonic duplex scanning. $J$ Vasc Surg 1986;4:450-6.

13 Bollinger A, Rutishauser W, Mahler F, Grüntzig A. Zur Dynamik des Rückstroms aus der Vena femoralis. Z Kreisl Forsch 1970;59:963-71.

14 Raghavendra BN, Roen RJ, Lam S, Riles T, Horii SC. Deep venous thrombosis: detection by high-resolution realtime ultrasonography. Radiology 1984;152:782-93.

15 Bollinger A, Mahler F, de Sepibus G. Diagnostik peripherer Venenerkrankungen mit Doppler-Strömungsdetektoren Disch Med Wochenschr 1968;93:2197-201.

16 Mahler F, Hirzel H, Bollinger A, Schönbeck M, Rutishauser W. Transkutane Registrierung der Strömungsgeschwindigkeit in der Vena jugularis mit Doppler-Strömungsdetektoren. Verh Dtsch Ges Kreisl-Forsch 1971;37:377-81 